\title{
CARIOTIPOS DE ESPECIES SUDAMERICANAS DE SERJANIA (SAPINDACEAE, PAULLINIEAE)
}

por VIVIANA G. SOLIS NEFFA ${ }^{1}$ Y MARIA S. FERRUCCI ${ }^{2}$

\section{Summary}

\begin{abstract}
Mitotic chromosomes of fifteen species of Serjania Mill., with $2 n=24$, from South America are studied. The results include the first counts for $S$. paludosa Cambess. and S. setigera Radlk. The karyotypes of all the species are analized, thirteen of them are described for the first time. Although there has been no change in chromosome number during the evolution of Serjania and the karyotypes show a moderated degree of asymmetry, there is considerable variability in chromosome size among the species. The comparative analysis of species of the same section shows differences in chromosome length and karyotype constitution. This was also evident in two accessions of $S$. laruotteana Cambess. and $S$. meridionalis Cambess. The karyotype variability observed in Serjania would be the result of structural chromosomic rearrangements andior the addition of genetic material.
\end{abstract}

\section{Introducción}

El género Serjania Mill. es el más numeroso de la familia, cuenta con alrededor de 230 especies de hábito trepador que son nativas de áreas tropicales y subtropicales del Nuevo Mundo. Radlkofer (1931) describió para el género doce secciones basadas en caracteres del fruto. Acevedo-Rodríguez (1993) propuso una reestructuración del género, fusionó ocho secciones en una sola y creó la sección Confertiflora.

Los estudios cromosómicos realizados en Serjania comprenden hasta el momento 24 especies, todas ellas con $2 n=2 x=24$ (AcevedoRodríguez, 1993; Fernández Casas \& Fernández Piqueras, 1981; Ferrucci, 1981, 1985; Ferrucci \& Solís Neffa, 1997; Guervin, 1961; Hemmer \& Morawetz, 1990; Maglioet al., 1984; Sarkar et al., 1982; Nogueira Z. et al., 1995) y, además, se ha descripto el cariotipo de $\delta$ de ellas (Hemmer \& Morawetz, 1990; Nogueira Z. et al., 1995).

${ }^{1}$ Instituto de Botánica del Nordeste (UNNE-CONICET), C.C. 209, (3400) Corrientes, Argentinâ.

${ }^{2}$ Niembro de la Carrera dei Investigador Científico y Tecnológı́co, CONICET. Instituto de Botánica del Nordeste.
En esta oportunidad se presenta el primer recuento cromosómico para $S$. paludosa y $S$. setigera, y se describen los cariotipos de quince especies, trece de ellos por primera vez. Este trabajo forma parte de un estudio citológico que las autoras están llevando a cabo sobre especies americanas de la familia, a fin de contribuir al esclarecimiento de problemas taxonómicos y evolutivos.

\section{Material y Métodos}

Los datos del material analizado se detallan en la Tabla 1; las especies se han ordenado según el sistema propuesto por Radlkofer (1931).

Se emplearon raíces de semillas germinadas en el laboratorio, pretratadas con 8-hidroxiquinoleína $2 \mathrm{mM}$ durante 3 horas a $20-22^{\circ} \mathrm{C}$, fijadas en etanol y ácido láctico $(5: 1)$ durante 12 horas y conservadas en alcohol $70 \%$ a $4^{\circ} \mathrm{C}$. La coloración se realizó siguiendo la técnica de Feulgen.

Se analizaron en promedio 10 metafases por especie, seleccionando las cinco mejores pará la confección de los idiogramas. Éstos fueron realizados sobre la base de las medias de las longitudes de los cromosomas y de los índices 
centroméricos (brazo corto x 100/ longitud total del cromosoma). La nomenclatura utilizada en la descripción de los cariotipos es la propuesta por Levan et al. (1964). Los símbolos m, $\mathrm{sm}$, st y $t$ designan respectivamente cromosomas metacéntricos, submetacéntricos, subtelocéntricos y acrocéntricos.

Se estimaron los siguientes parámetros para caracterizar numéricamente los cariotipos:

1. Longitud total del cariotipo (LTC)

2. Longitud cromosómica media (LM)

3. Índice centromérico promedio (IC)

4. Cociente entre la longitud par más largo/ par más corto ( $\mathrm{L} / \mathrm{C}$ )

5. Indice de asimetría intracromosómica $\left(A_{1}\right)=1-[\Sigma(b / B) / n]$

6. Índice de asimetría intercromosómica $\left(A_{2}\right)=s / x$

Las longitudes promedio de los brazos cortos y largos de cada par de cromosomas homólogos son b y B respectivamente; $\mathbf{n}$ es el número de pares de cromosomas homólogos; $s$ es la desviación típica y x la media de la longitud de los cromosomas.

La estimación de la asimetría de los cariotipos se realizó según el índice centromérico promedio, el cociente entre la longitud del par más largo/par más corto y según los índices $A_{1}$ y $A_{2}$ (Romero Zarco, 1986).

Los valores promedio de los parámetros calculados se compararon mediante análisis de la varianza (ANOVA) luego del test de Bartlett de homogeneidad de las varianzas. Las diferencias entre cada par de medias fueron estimadas mediante el test de Tukey.

\section{Resultados}

Los resultados se muestran en las Tablas 1-2 y en las Figuras 1-7.

Los cariotipos de las especies de Serjania analizadas están compuestos de doce pares de cromosomas, entre los que predominan los metacéntricos y submetacéntricos. Presentan un par de cromosomas acrocéntricos, a excepción de $S$. erecta, que posee dos pares y de $S$. hebecarpa, que no tiene ninguno (Fig. 4). En $S$. subimpunctata se observan en todas las células analizadas cromosomas con los centrómeros muy extendidos, en particular el par metacéntrico más grande (Fig. 1D).
Sólo en algunas metafases se observan cromosomas con constricciones secundarias y, a veces, la región organizadora nucleolar está tan extendida que resulta casi imposible ubicar a qué cromosomas corresponden los satélites (Fig. 2E). En algunas especies se cuentan de 1 a 3 pares con microsatélite, presentes tanto en el brazo corto como en el brazo largo de cromosomas submetacéntricos o subtelocéntricos. S. hebecarpa es la única especie en la que, además de microsatélite, se observa con frecuencia un par con macrosatélite (Fig. 2F).

El análisis de la varianza entre las medias de los distintos parámetros cromosómicos de las quince especies dio siempre resultados significativos (Tabla 2). Se observa en dicha tabla que los parámetros más variables son la longitud total del cariotipo y la longitud cromosómica media. Esta última varía desde $1.77 \mu \mathrm{m}$ en $\mathrm{S}$. clematidifolia hasta $3.64 \mu \mathrm{m}$ en S. meridionalis, siendo $2.42 \mu \mathrm{m}$ el promedio estimado para el total de las especies tratadas. El rango de variación y la longitud cromosómica media para cada una de las especies se indican en la Tabla 2 y se representan gráficamente en la Fig. 5. El test de Tukey al nivel de 5\% muestra que, de acuerdo con la longitud cromosómica media pueden establecerse cuatro grupos cuyas medias no difieren significativamente entre sí: Grupo I- LM $\leq 2 \mu \mathrm{m}$, Grupo II- LM = 2.01-2.50 $\mu \mathrm{m}$, Grupo III- LM $=2.51-3 \mu \mathrm{m}$ y Grupo IV$\mathrm{LM}>3 \mu \mathrm{m}$.

El índice centromérico promedio para el total de las especies $(\mathrm{IC}=32.79)$ corresponde a la categoría de submetacéntrico, variando desde 27.86 en S. cuspidata a 38.25 en S. hebecarpa. Esta última es la única especie cuyo índice centromérico promedio corresponde a la categoría de metacéntrico. Cuando se representan simultáneamente la longitud cromosómica media y el índice centromérico promedio en un gráfico de coordenadas, se observa que las especies de los grupos II y III presentan mayor variación en el índice centromérico promedio que las de los grupos I y IV; por otra parte, se observa una tendencia hacia la asimetría en las especies del grupo $\mathrm{V}$, con cromosomas más grandes (Fig. 7).

El cociente entre la longitud del par más largo/par más corto (L/C) da una estimación de la variación en la longitud de los 
Tabla 1. Especies estudiadas.

Especie

Código 2n Fig. Coleccionista y Procedencia

Sect. Platycoccus

S. cuspidata Cambess.

S. paludosa Cambess.

Sect. Ceratococcus

S. setigera Radlk.

Sect. Eurycoccus

S. glutinosa Radlk.

S. subimpunctata Radlk

cus $24 \quad 1 \mathrm{~A}$

pal $24^{*} 1 \mathrm{~B}$

set $24^{*} 1 \mathrm{C}$

glu 24

sub $24 \quad 1 D$

Sect. Eucoccus

S. caracasana (Jacq.) Willd.

S. larnotteana Cambess.

car $24 \quad 1 \mathrm{E}$

larl $24 \quad 1 \mathrm{~F}$

lar2 $24 \quad 1 G$

\section{Sect. Pachycoccus}

S. erecta Radlk.

S. marginata Casar.

mar

gla

24

2B

S. glabrata Kunth

Sect. Simococcus

S. clematidifolia Cambess. cle 2

Sect. Oococcus

S. perulacea Radlk.

Sect. Syncoccus

S. foveata Griseb.

S. hebecarpa Benth.

S.meridionalis Cambess.

$\begin{array}{lll}\text { per } & 24 & 2 \mathrm{C} \\ & 24\end{array}$

fov $24 \quad 2 \mathrm{D}-\mathrm{E}$

heb $24 \quad 2 \mathrm{~F}$

mer1 $24 \quad 2 \mathrm{G}$
Somner 719. Brasil, Rio de Janeiro (RBR).

Hatschbach 64991. Brasil, Minas Gerais (CTES, MBM).
Pott et al. 7818. Brasil, Mato Grosso do Sul (CPAP, CTES).

Vanni et al. 2775. Argentina, Misiones (ASU, CTES, FCQ, MBM, WIS).

Hatschbach et al. 57016. Brasil, Bahia (CTES, MBM).

\footnotetext{
* Primer recuento para la especie.
}

cromosomas de los complementos. Los valores de este parámetro oscilan entre 1.54 en $S$. marginata y 2.51 en $S$. hebecarpa, siendo esta última especie la que presenta la mayor diferencia en longitud entre ambos pares. Dado que este parámetro sólo tiene en cuenta los cromosomas extremos, se tomó también el índice $A_{2}$ que da una medida de la variación de las longitudes de todos los cromosomas. Éste tiende a 1 cuando la diferencia en longitud entre los cromosomas es mayor. El índice $A_{1}$ varía de 0 a 1 y tiende a 0 cuando los cromosomas tienden a ser metacéntricos.

En el diagrama de dispersión (Fig. 6), en el que se representa la asimetría debida a la relación entre brazos cromosómicos $\left(A_{1}\right)$ y la debida a la variación entre la longitud de los cromosomas $\left(\mathrm{A}_{2}\right)$, se observa que las especies ocupan una posición central, es decir, los cariotipos son moderadamente asimétricos y en general presentan diferencias graduales en tamaño entre los cromosomas en cada una de 
Tabla 2. Fórmula cariotípica, longitud total del complemento, longitud cromosómica media, grupo según la longitud cromosómica media, rango de variación de la longitud cromosómica, índice centromérico promedio, cociente entre la longitud del par más largo y el par más corto, índices de asimetría intracromosómica e intercromosómica.

\begin{tabular}{|c|c|c|c|c|c|c|c|c|c|}
\hline Especie & $\begin{array}{l}\text { Fórmula } \\
\text { Cariotípica }\end{array}$ & $\begin{array}{l}\text { LTC } \\
\mu \mathrm{m}\end{array}$ & $\begin{array}{l}\mathrm{LM} \\
\mu \mathrm{m}\end{array}$ & Grupo & $\begin{array}{c}\text { Rango de } \\
\text { Variación } \\
\mu \mathrm{m}\end{array}$ & IC & $\mathrm{L} / \mathrm{C}$ & $A_{1}$ & $\mathrm{~A}_{2}$ \\
\hline S. cuspidata & $\begin{array}{c}6 m+6 s m+10 s t \\
+2 t\end{array}$ & $\begin{array}{c}60.68 \\
\text { def }\end{array}$ & $\begin{array}{l}2.60 \\
\text { cde }\end{array}$ & III & $2.06-3.68$ & $\begin{array}{c}27.86 \\
\mathrm{e}\end{array}$ & $\begin{array}{c}1.73 \\
b c\end{array}$ & $\begin{array}{c}0.61 \\
\mathrm{a}\end{array}$ & $\begin{array}{l}0.23 \\
a b c\end{array}$ \\
\hline S. paludosa & $\begin{array}{c}10 m+10 s m+2 s t \\
+2 t\end{array}$ & $\begin{array}{l}62.82 \\
\text { bcde }\end{array}$ & $\begin{array}{l}2.63 \\
\text { bcde }\end{array}$ & III & $2.02-3.79$ & $\begin{array}{c}32.81 \\
\text { bcd }\end{array}$ & $\begin{array}{l}1.88 \\
a b c\end{array}$ & $\begin{array}{l}0.50 \\
\mathrm{abcd}\end{array}$ & $\begin{array}{l}0.22 \\
a b c\end{array}$ \\
\hline S. setigera & $\begin{array}{c}8 m+8 s m+6 s t \\
+2 t\end{array}$ & $\begin{array}{c}77.18 \\
a b\end{array}$ & $\begin{array}{c}3.24 \\
a b\end{array}$ & IV & $1.73-4.68$ & $\begin{array}{l}31.22 \\
\text { bcde }\end{array}$ & $\begin{array}{c}2.26 \\
a b\end{array}$ & $\begin{array}{l}0.54 \\
\text { abcd }\end{array}$ & $\begin{array}{c}0.25 \\
\mathrm{ab}\end{array}$ \\
\hline S. glutinosa & $\begin{array}{c}10 m+6 s m+6 s t \\
+2 t\end{array}$ & $\begin{array}{l}46.66 \\
\text { fgh }\end{array}$ & $\begin{array}{l}1.97 \\
\text { efg }\end{array}$ & I & $1.59-2.72$ & $\begin{array}{l}34.04 \\
\text { abcd }\end{array}$ & $\begin{array}{l}1.94 \\
a b c\end{array}$ & $\begin{array}{l}0.48 \\
\text { bcd }\end{array}$ & $\begin{array}{l}0.22 \\
a b c\end{array}$ \\
\hline S. subimpunctat & $\begin{array}{c}t a 12 m+8 s m+2 s t \\
+2 t\end{array}$ & $\begin{array}{c}44.02 \\
h\end{array}$ & $\begin{array}{l}1.84 \\
\text { gh }\end{array}$ & I & $1.31-2.63$ & $\begin{array}{c}35.30 \\
a b\end{array}$ & $\begin{array}{l}2.01 \\
\mathrm{abc}\end{array}$ & $\begin{array}{l}0.45 \\
\text { de }\end{array}$ & $\begin{array}{l}0.21 \\
a b c\end{array}$ \\
\hline S. caracasana & $\begin{array}{c}8 m+10 s m+4 s t \\
+2 t\end{array}$ & $\begin{array}{l}62.60 \\
\text { cde }\end{array}$ & $\begin{array}{l}2.60 \\
\text { cde }\end{array}$ & III & $2.05-3.71$ & $\begin{array}{l}32.22 \\
\text { bcde }\end{array}$ & $\begin{array}{l}1.73 \\
b c\end{array}$ & $\begin{array}{l}0.52 \\
a b c\end{array}$ & $\begin{array}{l}0.22 \\
a b c\end{array}$ \\
\hline \multicolumn{10}{|l|}{ S. laruotteana } \\
\hline F. 843 & $\begin{array}{c}10 m+8 s m+4 s t \\
+2 t\end{array}$ & $\begin{array}{l}61.66 \\
\text { cdef }\end{array}$ & $\begin{array}{l}2.64 \\
\text { bcde }\end{array}$ & III & $2.13-3.50$ & $\begin{array}{l}34.50 \\
\text { abcd }\end{array}$ & $\begin{array}{l}1.73 \\
b c\end{array}$ & $\begin{array}{l}0.46 \\
\text { bcde }\end{array}$ & $\begin{array}{c}0.20 \\
b c\end{array}$ \\
\hline A. 5926 & $\begin{array}{c}10 m+8 s m+4 s t \\
+2 t\end{array}$ & $\begin{array}{l}44.68 \\
\text { gh }\end{array}$ & $\begin{array}{l}1.85 \\
\mathrm{fgh}\end{array}$ & I & $1.38-2.68$ & $\begin{array}{c}34.84 \\
\mathrm{abc}\end{array}$ & $\begin{array}{l}2.01 \\
a b c\end{array}$ & $\begin{array}{l}0.46 \\
\text { cde }\end{array}$ & $\begin{array}{l}0.23 \\
a b c\end{array}$ \\
\hline S. erecta & $\begin{array}{c}8 m+8 s m+4 s t \\
+4 t\end{array}$ & $\begin{array}{l}61.96 \\
\text { cde }\end{array}$ & $\begin{array}{c}2.60 \\
\mathrm{e}\end{array}$ & III & $1.83-3.56$ & $\begin{array}{l}30.83 \\
\text { cde }\end{array}$ & $\begin{array}{l}1.95 \\
\mathrm{abc}\end{array}$ & $\begin{array}{l}0.55 \\
a b c d\end{array}$ & $\begin{array}{l}0.23 \\
a b c\end{array}$ \\
\hline S. marginata & $\begin{array}{c}8 m+8 s m+6 s t \\
+2 t\end{array}$ & $\begin{array}{l}74.50 \\
\text { abcd }\end{array}$ & $\begin{array}{l}3.09 \\
\text { abcd }\end{array}$ & IV & $2.33-4.10$ & $\begin{array}{l}30.52 \\
\text { cde }\end{array}$ & $\begin{array}{c}1.54 \\
c\end{array}$ & $\begin{array}{l}0.56 \\
a b c\end{array}$ & $\begin{array}{l}0.21 \\
a b c\end{array}$ \\
\hline S. glabrata & $\begin{array}{c}8 \mathrm{~m}+10 \mathrm{sm}+4 \mathrm{st} \\
+2 \mathrm{t}\end{array}$ & $\begin{array}{l}75.60 \\
a b c\end{array}$ & $\begin{array}{l}3.15 \\
a b c\end{array}$ & IV & $2.35-4.16$ & $\begin{array}{l}32.10 \\
\text { bcde }\end{array}$ & $\underset{c}{1.55}$ & $\begin{array}{l}0.52 \\
\text { abcd }\end{array}$ & $\begin{array}{l}0.23 \\
a b c\end{array}$ \\
\hline S. clematidifolia & $\begin{array}{c}8 m+12 s m+2 s t \\
+2 t\end{array}$ & $\begin{array}{c}42.56 \\
\mathrm{~h}\end{array}$ & $\begin{array}{c}1.77 \\
\mathrm{~h}\end{array}$ & I & $1.30-2.25$ & $\begin{array}{l}34.16 \\
\text { abcd }\end{array}$ & $\begin{array}{c}1.61 \\
b c\end{array}$ & $\begin{array}{l}0.48 \\
\text { bcd }\end{array}$ & $\begin{array}{c}0.19 \\
b c\end{array}$ \\
\hline S. perulacea & $\begin{array}{c}8 \mathrm{~m}+8 \mathrm{sm}+6 \mathrm{st} \\
+2 \mathrm{t}\end{array}$ & $\begin{array}{l}54.40 \\
\text { efgh }\end{array}$ & $\begin{array}{l}2.28 \\
\text { efgh }\end{array}$ & II & $1.73-3.06$ & $\begin{array}{l}31.25 \\
\text { bcde }\end{array}$ & $\begin{array}{c}1.70 \\
b c\end{array}$ & $\begin{array}{l}0.54 \\
a b c d\end{array}$ & $\begin{array}{l}0.22 \\
a b c\end{array}$ \\
\hline S. foveata & $\begin{array}{c}8 m+12 s m+2 s t \\
+2 t\end{array}$ & $\begin{array}{l}60.58 \\
\text { def }\end{array}$ & $\begin{array}{l}2.53 \\
\text { cdef }\end{array}$ & II & $2.07-3.25$ & $\begin{array}{l}33.92 \\
\text { abcd }\end{array}$ & $\begin{array}{c}1.57 \\
c\end{array}$ & $\begin{array}{l}0.48 \\
\text { bcd }\end{array}$ & $\begin{array}{c}0.19 \\
c\end{array}$ \\
\hline 5. hebecarpa & $16 m+2 s m+6 s t$ & $\begin{array}{l}59.10 \\
\text { efg }\end{array}$ & $\begin{array}{l}2.47 \\
\text { defg }\end{array}$ & II & $1.59-3.66$ & $\begin{array}{c}38.25 \\
\mathrm{a}\end{array}$ & $\begin{array}{c}2.51 \\
\mathrm{a}\end{array}$ & $\begin{array}{c}0.37 \\
\mathrm{e}\end{array}$ & $\begin{array}{c}0.27 \\
\mathrm{a}\end{array}$ \\
\hline \multicolumn{10}{|l|}{ S.meridionalis } \\
\hline R. 52 & $\begin{array}{c}6 m+10 s m+6 s t \\
+2 t\end{array}$ & $\begin{array}{c}86.06 \\
a\end{array}$ & $\begin{array}{c}3.64 \\
a\end{array}$ & IV & $2.60-4.73$ & $\begin{array}{c}29.78 \\
\text { de }\end{array}$ & $\begin{array}{l}2.07 \\
a b c\end{array}$ & $\begin{array}{c}0.57 \\
\mathrm{ab}\end{array}$ & $\begin{array}{l}0.23 \\
a b c\end{array}$ \\
\hline R. 53 & $\begin{array}{c}8 m+12 s m+2 s t \\
+2 t\end{array}$ & $\begin{array}{l}46.70 \\
\text { efgh }\end{array}$ & $\begin{array}{l}2.10 \\
\text { efgh }\end{array}$ & II & $1.44-2.81$ & $\begin{array}{l}33.89 \\
\text { abcd }\end{array}$ & $\begin{array}{l}1.91 \\
a b c\end{array}$ & $\begin{array}{l}0.48 \\
\text { bcd }\end{array}$ & $\begin{array}{l}0.22 \\
a b c\end{array}$ \\
\hline F (ANOVA) & & $19.81^{* *}$ & $16.89^{* *}$ & & & $7.82^{* *}$ & $3.83^{* *}$ & $7.99^{* *}$ & $2.52^{* *}$ \\
\hline
\end{tabular}

${ }^{* *} \mathrm{p}<.01$

Nota: Los valores de la misma columna seguidos de la misma letra no presentan diferencias significativas. Abreviaturas ver Material y Métodos.

las especies. S. hebecarpa posee el cariotipo más simétrico $\left(\mathrm{A}_{1}=0.37\right)$ y es la especie que presenta la mayor variación de longitud entre sus cromosomas $\left(\mathrm{A}_{2}=0.27\right)$. S. cuspidata es la especie con el cariotipo más asimétrico $\left(A_{1}=0.61\right)$, mientras que $S$. clematidifolia y $S$. foveata pre- 
Tabla 3. Estudios cromosómicos realizados en Serjania por diversos autores.

\begin{tabular}{|c|c|c|c|c|c|}
\hline Sección & Especie & $2 n$ & $\begin{array}{l}\text { Fórmula } \\
\text { Cariotípica }\end{array}$ & Grupo & Autor \\
\hline \multirow[t]{4}{*}{ Platycoccus } & S. communis Cambess. & 24 & $6 m+18 s m$ & II & Nogueira Z. et al. (1995) \\
\hline & S. confertiflora Radlk. & 24 & - & - & $\begin{array}{l}\text { Fernández C. \& Fernández } \\
\text { P. (1981) }\end{array}$ \\
\hline & S. cuspidata & 24 & $6 m+6 s m+10 s t+2 t$ & III & Este trabajo \\
\hline & S. paludosa & 24 & $10 m+10 s m+2 s t+2 t$ & III & Este trabajo \\
\hline Ceratococcus & S. setigera & 24 & $8 m+8 s m+6 s t+2 t$ & IV & Este trabajo \\
\hline \multirow[t]{6}{*}{ Eurycoccus } & $\begin{array}{l}\text { S. diversifolia (Jacq.) } \\
\text { Radlk. }\end{array}$ & 24 & $8 m+14 s m+2 t$ & II & $\begin{array}{l}\text { Hemmer \& Morawetz } \\
(1990)\end{array}$ \\
\hline & S. gracilis Radlk. & 24 & $14 m+6 s m+4 s t$ & II & Nogueira Z. et al. (1995) \\
\hline & & 24 & $12 m+8 s m+4 s t$ & I & Nogueira Z. et al. (1995) \\
\hline & & 24 & $12 \mathrm{~m}+12 \mathrm{sm}$ & III & Nogueira Z. et al. (1995) \\
\hline & S. glutinosa & 24 & $10 m+6 s m+6 s t+2 t$ & I & Este trabajo \\
\hline & S. subimpunctate $\tilde{c}_{i}$ & 24 & $12 m+8 s m+2 s t+2 t$ & I & Este trabajo \\
\hline \multirow[t]{5}{*}{ Eucoccus } & S. caracasana & 24 & $8 m+10 s m+4 s t+2 t$ & III & Este trabajo \\
\hline & S. lariotteana & 24 & $18 m+2 s m+4 s t$ & II & Nogueira Z. et al. (1995) \\
\hline & & 24 & $10 \mathrm{~m}+10 \mathrm{sm}+4 \mathrm{st}$ & I & Nogueira Z. et al. (1995) \\
\hline & & 24 & $10 m+8 s m+4 s t+2 t$ & III & Este trabajo \\
\hline & & 24 & $10 m+8 s m+4 s t+2 t$ & I & Este trabajo \\
\hline \multirow[t]{2}{*}{ Pachycoccus } & S. erecta & 24 & $8 m+8 s m+4 s t+4 t$ & IIII & Este trabajo \\
\hline & S. marginata & 24 & $8 m+8 s m+6 s t+2 t$ & IV & Este trabajo \\
\hline \multirow[t]{4}{*}{ Holcococcus } & S. exarata Radlk. & 24 & - & - & Guervin (1961) \\
\hline & S. glabrata & 24 & $8 m+10 s m+4 s t+2 t$ & IV & Este trabajo \\
\hline & & 24 & $10 m+12 s m+2 s t$ & III & Nogueira Z. et al. (1995) \\
\hline & S. tripleviria Ferrucci & 24 & - & - & Ferrucci (1985) \\
\hline Serjania & S. subdentata Juss. & 24 & $6 m+16 s m+2 t$ & II & $\begin{array}{l}\text { Hemmer \& Morawetz } \\
\text { (1990) }\end{array}$ \\
\hline$(=$ Dictyococcus $)$ & S. lucida Schumach. & 24 & - & - & Guervin (1961) \\
\hline \multirow[t]{3}{*}{ Simococcus } & S. clematidifolia & 24 & $8 m+12 s m+2 s t+2 t$ & I & Este trabajo \\
\hline & S. fuscifolia Radlk. & 24 & $16 \mathrm{~m}+8 \mathrm{sm}$ & I & Nogueira Z. et al. (1995) \\
\hline & & 24 & $18 m+4 s m+2 s t$ & II & Nogueira Z. et al. (1995) \\
\hline \multirow[t]{2}{*}{ Oococcus } & S. multiflora Cambess. & 24 & $10 \mathrm{~m}+14 \mathrm{sm}$ & III & Nogueira Z. et al. (1995) \\
\hline & S. perulacea & 24 & $8 m+8 s m+6 s t+2 t$ & II & Este trabajo \\
\hline \multirow{2}{*}{$\begin{array}{l}\text { Phacococcus } \\
\text { Physococcus }\end{array}$} & & & sin datos & & \\
\hline & & & sin datos & & \\
\hline \multirow[t]{5}{*}{ Syncoccus } & S. cissoides Radlk. & 24 & - & - & Ferrucci (1981) \\
\hline & S. foveata & 24 & $8 m+12 s m+2 s t+2 t$ & II & Este trabajo \\
\hline & S. hebecarpa & 24 & $16 m+2 s m+6 s t$ & II & Este trabajo \\
\hline & S. meridionalis & 24 & $6 m+10 s m+6 s t+2 t$ & IV & Este trabajo \\
\hline & & 24 & $8 m+12 s m+2 s t+2 t$ & II & Este trabajo \\
\hline
\end{tabular}

sentan la menor variación entre las longitudes de sus cromosomas $\left(A_{2}=0.19\right)$.

\section{Discusión}

Los estudios cromosómicos realizados hasta el momento en Serjania comprenden recuentos cromosómicos de especies pertenecientes a nueve de las doce secciones con que cuenta el género y el cariotipo de ocho especies distribuidas en siete secciones. El recuento realizado en $S$. setigera es el primero para la sección Ceratococcus, los cariotipos de $S$. erecta y $S$. marginata constituyen las primeras descripciones para la sección Pachycoccus, y los de $S$. 
foveata, S. hebecarpa y S. meridionalis para Syncoccus (Tabla 3).

En Sapindaceae la aneuploidía tuvo un papel importante en la evolución cariotípica, siendo la poliploidía muy poco frecuente (Ferrucci, 1981; Hemmer \& Morawetz, 1990; Nogueira Z. et al., 1995; Ferrucci \& Solís Neffa, 1997). En Serjania, aunque sólo se tienen datos para el $13 \%$ de las especies, la constancia del número cromosómico es evidente. Entre las especies y dentro de las mismas existen diferencias en las respectivas fórmulas cariotípicas, en el tamaño de los cromosomas, así como en el grado de asimetría de los cariotipos.

Las especies revisadas presentan cariotipos en los que predominan los cromosomas metacéntricos y submetacéntricos, acompañados por algunos pares subtelocéntricos y acrocéntricos. Hemmer \& Morawetz (1990) estudiaron los cariotipos de S. subdentata y $S$. diversifolia, aunque no hicieron un análisis numérico detallado de los mismos y tampoco citan la procedencia del material; los describieron como constituidos por cromosomas metacéntricos, submetacéntricos y un par acrocéntrico. Nogueira Z. et al. (1995) analizaron los cariotipos de seis especies de Serjania y determinaron que los mismos poseen cromosomas metacéntricos y submetacéntricos; sólo para tres especies citaron cromosomas subtelocéntricos. Nogueira Z. et al. (1995) describieron los cariotipos de dos accesiones de $S$. laruotteana, una de ellas con $18 m+2 s m+4 s t$ y la otra con $10 m+10 s m+4 s t$, y también el cariotipo de S. glabrata, $10 \mathrm{~m}+12 \mathrm{sm}+2 \mathrm{st}$. En el presente trabajo se estudiaron dos accesiones de S. laruotteana que poseen igual cariotipo, $10 m+8 s m+4 s t+2 t$ y $S$. glabrata con $8 m+10 s m+4 s t+2 t$. Estos cariotipos se diferencian del material analizado por Nogueira Z. et al. (1995) porque poseen cromosomas acrocéntricos.

En S. subimpunctata se identificaron cromosomas con el centrómero extendido; este tipo de centrómero registra antecedentes en géneros de otras familias (Lima de Faría, 1956; Hunziker, 1961; Fernández \& Krapovickas, 1994).

Respecto al tipo y número de satélites, nuestros resultados concuerdan con observaciones previas de otros autores. Los satélites son difí- ciles de observar; las especies analizadas muestran de 1 a 3 pares con microsatélite, excepto $S$. hebecarpa en la que también se observa un par de cromosomas con macrosatélite. Hemmer \& Morawetz (1990) observaron hasta tres pares de cromosomas con microsatélite en $S$. subdentata y $S$. diversifolia, mientras que Nogueira Z. et al. (1995) encontraron dos pares en S. laruotteana y un par en S. communis. En el material de S. laruotteana analizado en este trabajo se observaron dos pares con microsatélite en la accesión F. 843, y ninguno en la accesión A. 5926.

Nogueira Z. et al. (1995) emplearon técnicas de tinción con nitrato de plata (Ag-NOR) para una mejor caracterización de los cariotipos de Serjania. En la mayoría de los casos, el número de regiones organizadoras nucleolares activas (NORs) concuerda con el número máximo de nucléolos. En S. laruotteana observaron dos pares con microsatélite y hasta cuatro nucléolos, mientras que en S. communis detectaron un par con satélite y hasta cinco nucléolos. Estos autores establecieron tres grupos de acuerdo con el número de nucléolos observados, siendo seis el número máximo detectado, es decir, hasta tres pares de cromosomas con satélite. Los autores citados también señalaron que en algunas especies el número máximo de nucléolos puede variar entre las diferentes accesiones estudiadas mientras que en otras éste permanece constante.

Respecto del tamaño, los cromosomas de Serjania corresponden a la categoría de pequeños (1-4 $\mu \mathrm{m})$ y medianos (4-12 $\mu \mathrm{m})$, según la clasificación de Lima de Faría (1980) y éste es el parámetro analizado que presenta mayor variabilidad entre las especies. Nuestros resultados apoyarían lo propuesto por Hemmer \& Morawetz (1990) quienes sugirieron que en la tribu Paullinieae el aumento en la longitud de los cromosomas pudo actuar como una alternativa a la poliploidización. Los mencionados autores observaron que en $S$. diversifolia y en $S$. subdentata el rango de variación en longitud cromosómica es 1.7-3 $\mu \mathrm{m}$, mientras que Nogueira Z et al. (1995) citan $1.36 \mu \mathrm{m}$ en S. gracilis y $2.52 \mu \mathrm{m}$ en $S$. glabrata.

Los cariotipos en Serjania son moderadamente asimétricos y presentan diferencias graduales en el tamaño de sus cromosomas; se 


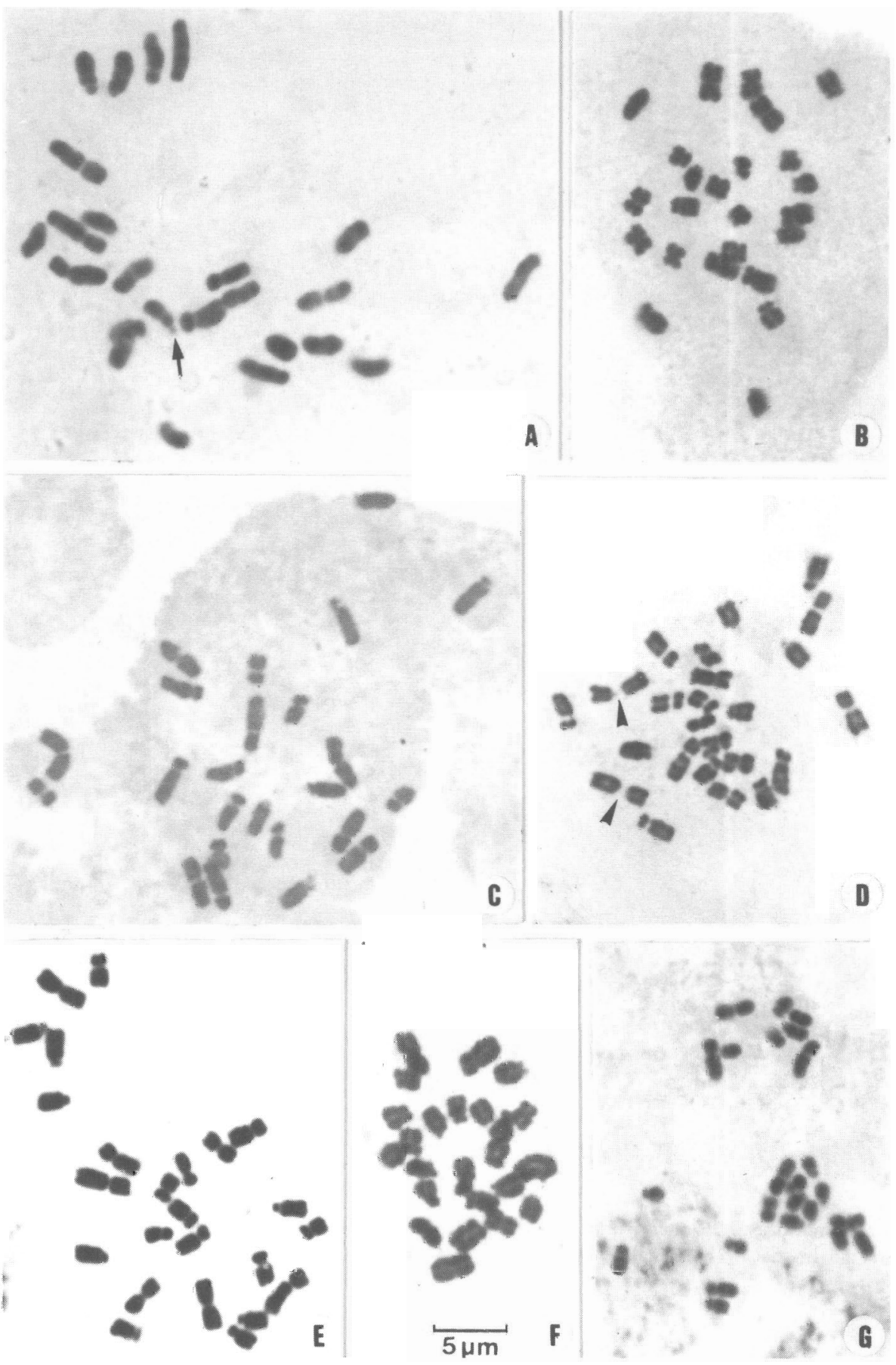

Fig.1. Metafases mitóticas. A, Serjania cuspidata; B, S. paludosa; C, S. setigera; D, S. subinupunctata; E, S. caracasana; F, S. laruotteana (F. 843); G, S. laruotteana (A. 5926). Escala= $5 \mu \mathrm{m}$. $\uparrow$ señalan cromosomas con satélite, A centrómeros extendidos.

observa una tendencia hacia la asimetría en las especies con cromosomas más grandes.

La información cariológica sobre Serjania obtenida de distintos autores se resume en la
Tabla 3. En la misma se observa que en las secciones donde se estudiaron dos o más especies e, inclusive, en las entidades en las que se analizó más de una accesión, los cariotipos pre- 


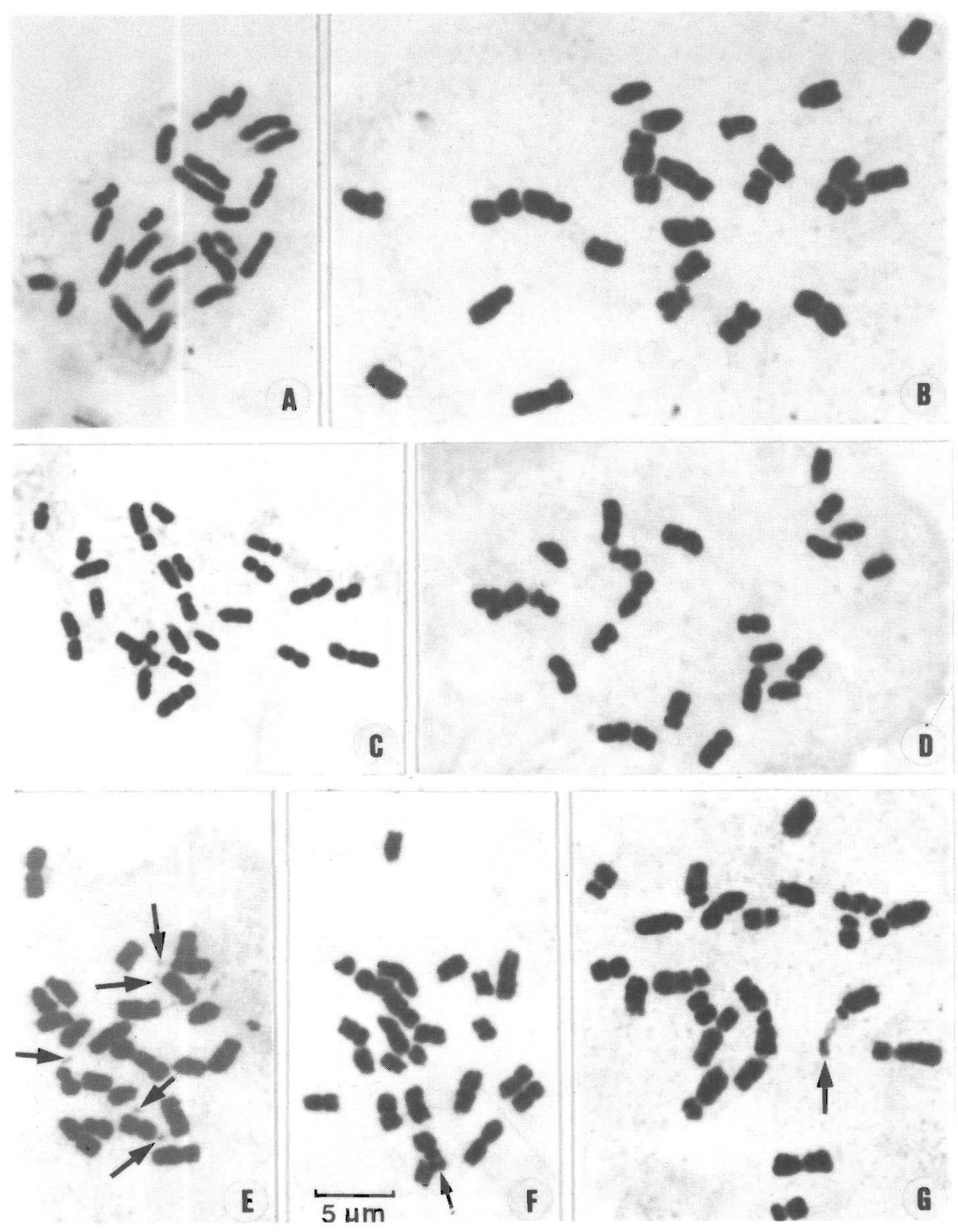

Fig. 2. Metafases mitóticas. A, Serjania erecta; B, S. glabrata; C, S. peruiacea; D-E, S. foveata; F, S. hebecarpa; G, S. meridionalis (R. 52). Escala $=5 \mu \mathrm{m}$. Las flechas señalan satélites.

sentan diferencias. Dentro de las secciones, las especies aunque difieren en su fórmula cariotípica muestran poca variación en la longitud cromosómica media, siendo la sección Eucoccus la que presenta la mayor variación. Las accesiones de $S$. laruotteana y las de $S$. meridonalis muestran distintas longitudes cromosómicas medias; además, en esta última especie se observaron variaciones en su fórmu- la cariotípica. Estos resultados coinciden con los de Nogueira Z. et al. (1995), quienes también encontraron diferencias en S. gracilis, $S$. laruotteana y en $S$. fuscifolia, especies en las que estudiaron más de una accesión.

La información citológica es aún escasa y no se tienen datos de las secciones Phacococcus y Physococcus. Sin embargo, la constancia en el numero cromosómico, la variación en las lon- 
S. cuspidata

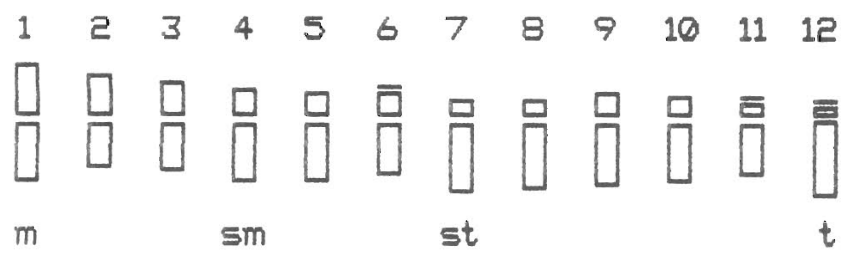

S. paludosa

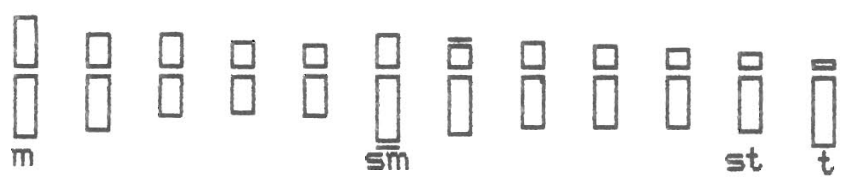

S. setigera

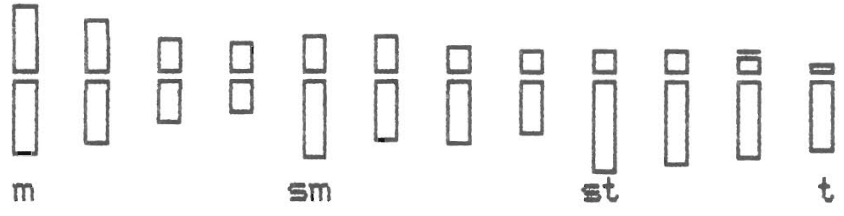

S. glutingsa

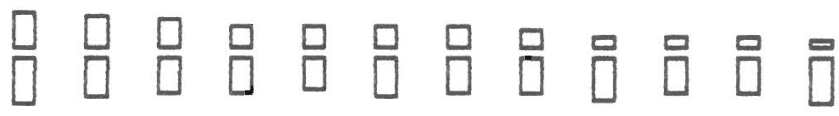

S. subimpunctata

m

$\begin{array}{llll}\square & \square & \square & \square\end{array}$

m

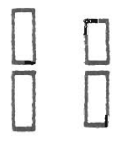

S. laruottiana m

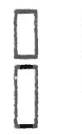
F. 843 A. 5926

S. caracasana

S. Laruotteana

m

$\begin{array}{lllll}\square & \square & \square & \square & \square\end{array}$

m
$5 \mathrm{~m}$

st
吕吕吕

sm

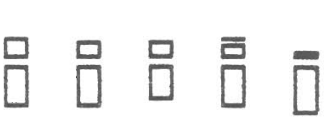

st $t$

Fig. 3. Idiogramas de Serjania. Escala $=5 \mu \mathrm{m}$.

gitudes cromosómicas medias y las diferencias observadas en la simetría de los cariotipos, aún en diferentes accesiones de una misma especie, podrían ser el resultado de cambios estructurales y/o la adición del material genético.

\section{Agradecimientos}

Deseamos expresar nuestro agradecimiento al Ing. Agr. A. Fernández por el asesoramiento y la lectura crítica del manuscrito y al Sr. A. 
S. erecta

$\begin{array}{llllllllllll}1 & 2 & 3 & 4 & 5 & 6 & 7 & 8 & 9 & 10 & 11 & 12\end{array}$

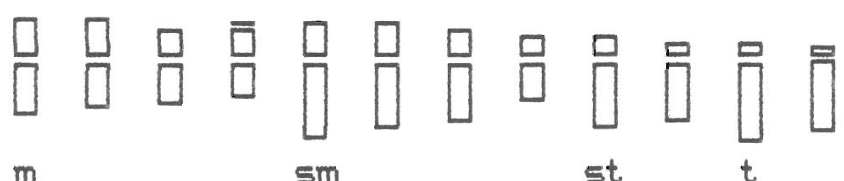

S. marginata

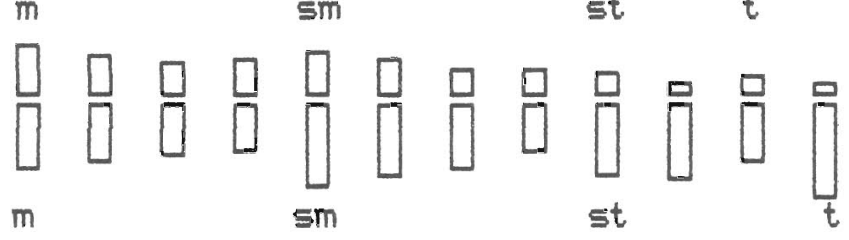

5. glabrata

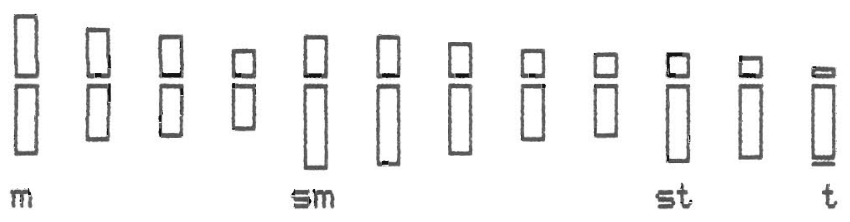

S. clematidifolia

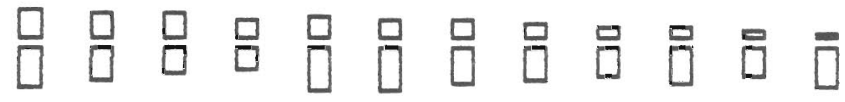
$m \quad s m$ st $t$

S. perulacea

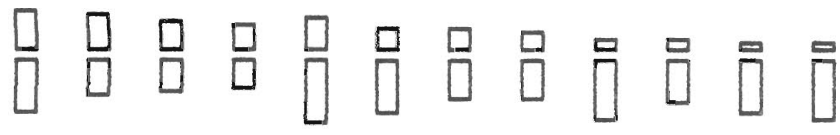

m

$5 \mathrm{~m}$

st

$\begin{array}{lllllllllll}\square & \square & \square & \square & \square & \square & \square & \square & \square & \square & \square\end{array}$

$m$

sm

st $t$

S. hebecarpa

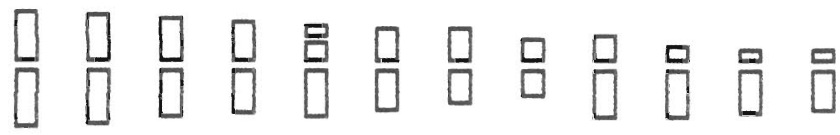

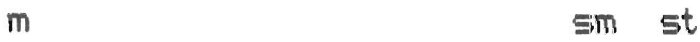

S. meridionalis R. 52

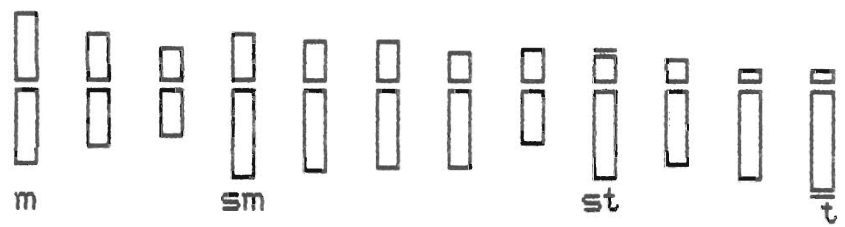

S. meridionalis R. 53

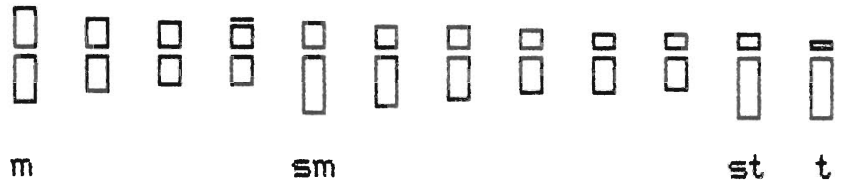

Fig. 4. Idlogramas de Serjania. Escaia $=5 \mu \mathrm{m}$ 


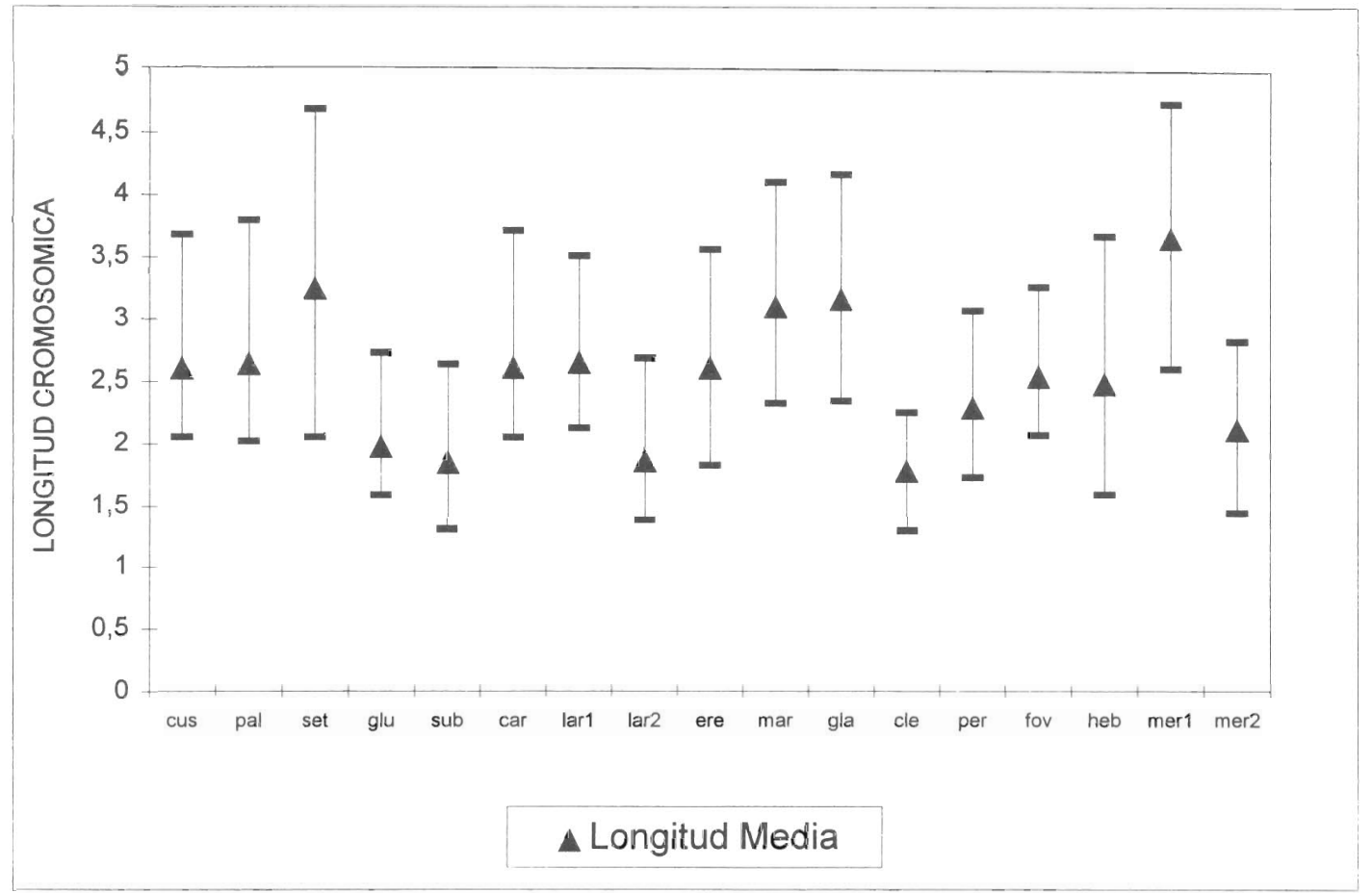

Fig. 5. Diagrama que representa el rango de variación en longitud de los cromosomas de las especies estudiadas. Los valores están expresados en $\mu \mathrm{m}$ y se detallan en la Tabla 2. Los códigos figuran en la Tabla 1.

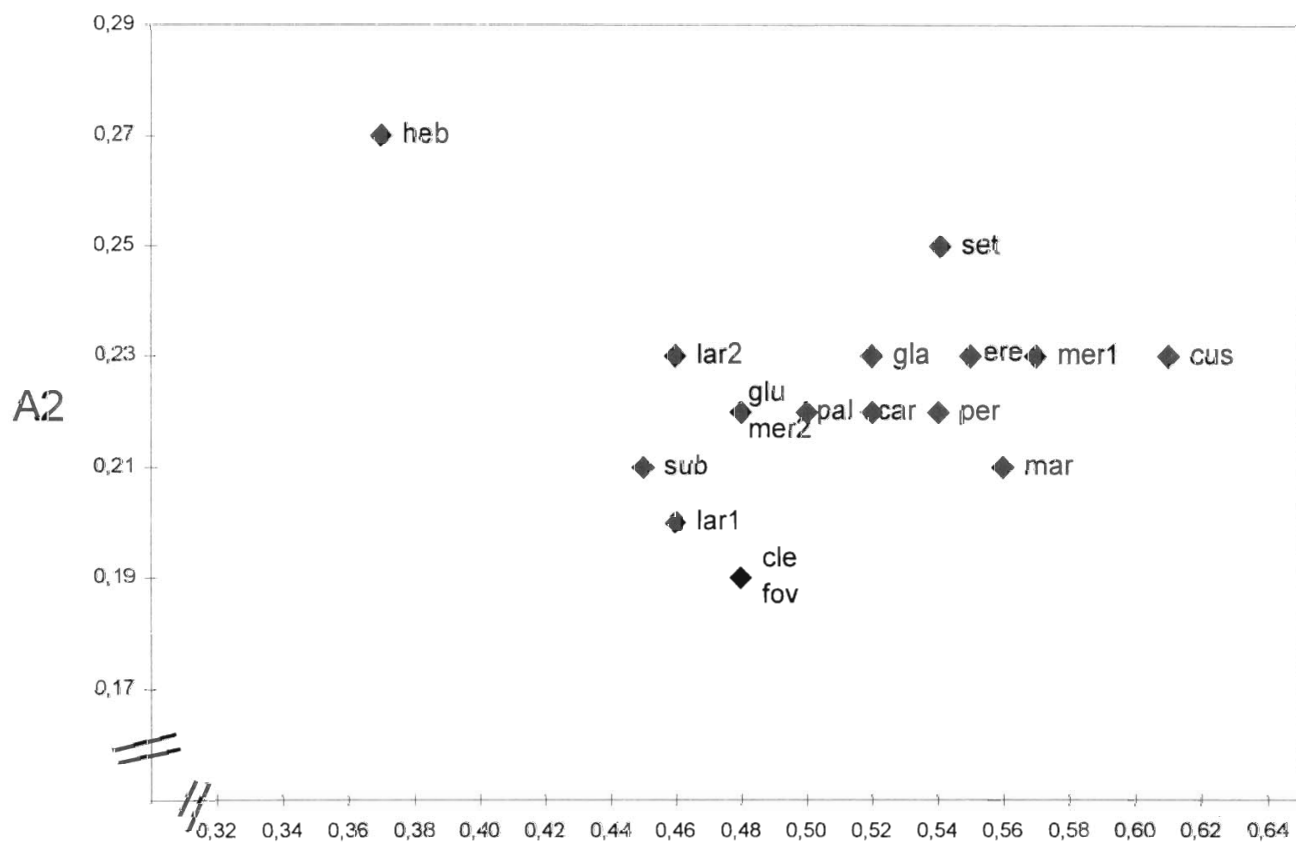

A1

Fig. 6. Diagrama de dispersión que representa la asimetría debida a la relación entre brazos cromosómicos $\left(\mathrm{A}_{1}\right)$ y la debida a la variación entre la longitud de los cromosomas $\left(\mathrm{A}_{2}\right)$. Los valores de $\mathrm{A}_{1}$ y $\mathrm{A}_{2}$ se detallan en la Tabla 2. Los códigos figuran en la Tabla 1. 


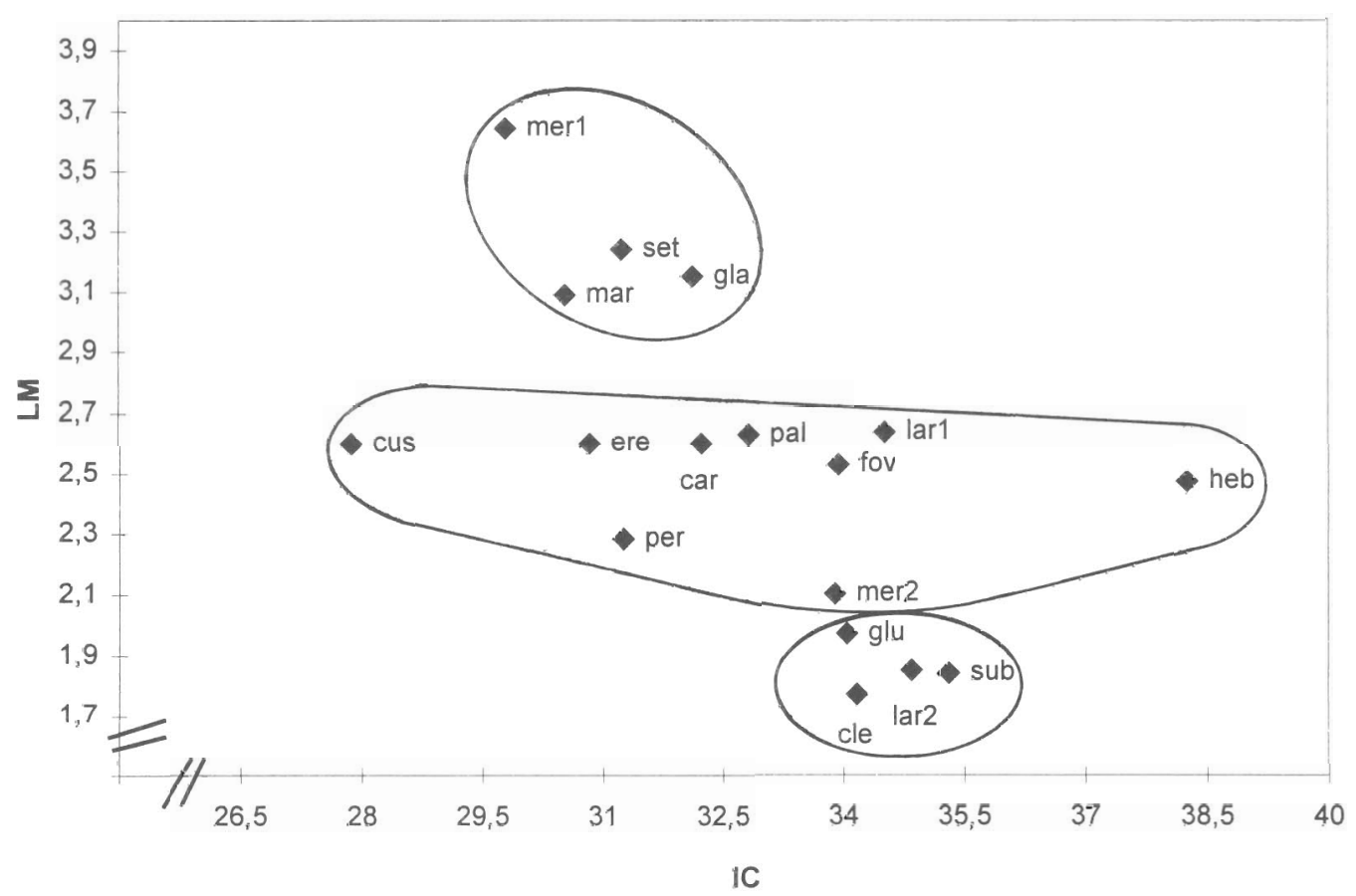

Fig. 7. Diagrama de dispersión que representa la relación entre la longitud cromosómica media (LM) y el índice centromérico promedio (IC). Los valores de LM e IC se detallan en la Tabla 2. Los códigos figuran en la Tabla 1.

Graciani por su colaboración en la confección de los gráficos. Este trabajo se llevó a cabo con el apoyo económico de la Secretaría General de Ciencia y Técnica (UNNE) y del subsidio \# 5343-94 de la National Geographic Society.

\section{Bibliografía}

ACEVEDO-RODRÍGUEZ, P. 1993. Systematics of Serjania (Sapindaceae). Part I: A revision of Serjania Sect. Platycoccus. Mem. New York Bot. Gard. 67: 1-93.

FERNÁNDEZ, A.\&A. KRAPOVICKAS. 1994. Cromosomas y Evolución en Arachis (Leguminosae). Bonplandia 8 (14): $187-220$.

FERNÁNDEZ CASAS, J. \& J. FERNÁNDEZ PIQUERAS. 1981. Estudio cariológico de algunas plantas bolivianas. Anales Jard. Bot. Madrid 38 (1): 149-152.

FERRUCCI, M.S. 1981. Recuentos cromosómicos en Sapindáceas. Bonplandia 5 (11): 73-81.

- 1985. Recuentos cromosómicos en Allophylus y Serjania (Sapindaceae). Bol. Soc. Argent. Bot. 24 (1-2): 200-202.

FERRUCCI, M.S. \& V. G. SOLÍS NEFFA. 1997. Citotaxonomía deSapindaceae Sudamericanas. Bol. Soc. Argent. Bot. 33 (1-2). En prensa.

GUERVIN, C. 1961. Contribution à l' étude cytotaxinomique des Sapindacées et caryologique des Mélianthacées et des Didiéréacées. Rev. Cytol. Biol. Vég. 23 (1): 49-87.
HEMMER, W. \& W. MORAWETZ. 1990. Karyological differentiation in Sapindaceae with special reference to Serjania and Cardiospermum. Bot. Acta 103(4): 372-383.

HUNZIKER,J.H. 1961. Estudios cromosómicos en Cupressus y Libocedrus (Cupressaceae). Revista Invest. Agric. 15 (2): $169-185$

LEVAN, A., K. FREDGA \& A. A. SANDBERG. 1964. Nomenclature for centromeric position on chromosomes. Hereditas 52: 201-220.

LIMA DE FARÍA, A. 1956. The role of the kinetochore in chromosome organization. Hereditas 42: 85-160.

- 1980. Classification of genes, rearrangements and chromosomes according to the field. Hereditas 93: 146.

MAGLIO, C.A.F.P., E.R. FORNI-MARTINS \& N.D. DA CRUZ. 1984. En: Löve, A. (ed.), Chromosome number reports LXXXIV. Taxon 33 (3): 536.

NOGUEIRA ZAMPIERI, C., P.M. RUAS, C.F. RUAS \& M.S. FERRUCCI. 1995. Karyotypic study of some species of Serjania and Urvillea (Sapindaceae; Tribe Paullinieae). Amer. J. Bot. 82 (5): 646-654.

RADLKOFER, L. 1931.Sapindaceae, in: Engler, A. Pflanzenr. 98 (IV. 165): 19-219.

ROMERO ZARCO, C. 1986. A new method for estimating karyotype asymmetry. Taxon 35: 526-530.

SARKAR, A.K., N. DATTA, U. CHATTERJEE\&D. HAZRA. 1982. In: Löve, A. (ed.), IOPB Chromosome number reports LXXVI. Taxon 31 (3): 578. 